\title{
CURVATURE BLOW UP ON A DENSE SUBSET OF THE SINGULARITY IN $T^{3}$-GOWDY
}

\author{
HANS RINGSTRÖM
}

\begin{abstract}
This paper is concerned with the Einstein vacuum equations under the additional assumption of $T^{3}$-Gowdy symmetry. We prove that there is a generic set of initial data such that the corresponding solutions exhibit curvature blow up on a dense subset of the singularity. By generic, we mean a countable intersection of open sets (i.e. a $G_{\delta}$ set) which is also dense. Furthermore, the set of initial data is given the $C^{\infty}$ topology. This result was presented at a conference in Miami 2004. Recently, we have obtained a stronger result, but the argument to prove it is different and much longer. Therefore, we here wish to present the original argument. Finally, combining the results presented here with a paper by Chruściel and Lake, one obtains strong cosmic censorship for $T^{3}$-Gowdy spacetimes.
\end{abstract}

\section{INTRODUCTION}

The motivation for studying the problem discussed in this paper is the desire to understand the structure of singularities in cosmological spacetimes. By the singularity theorems, cosmological spacetimes typically have a singularity in the sense of causal geodesic incompleteness. However, it seems that the methods used to obtain this result are not so well suited to answering related questions concerning e.g. curvature blow up. To proceed, it seems difficult to avoid analyzing the equations in detail. After some appropriate choice of gauge, one is then confronted with the task of analyzing the asymptotics of a non-linear hyperbolic equation. Since this is difficult in general, one often imposes some symmetry condition, and we shall here consider a class of spacetimes with a two dimensional group of symmetries. The problem one ends up with is then a system of non-linear wave equations in $1+1$ dimensions.

The Gowdy spacetimes were first introduced in [7] (see also [5]), and in [11] the fundamental questions concerning global existence were answered. We shall take the Gowdy vacuum spacetimes on $\mathbb{R} \times T^{3}$ to be metrics of the form (1). For natural geometric conditions leading to this form of the metric, we refer the reader to [7] and [5] or, for a brief description, [14]. Let

(1) $g=e^{(\tau-\lambda) / 2}\left(-e^{-2 \tau} d \tau^{2}+d \theta^{2}\right)+e^{-\tau}\left[e^{P} d \sigma^{2}+2 e^{P} Q d \sigma d \delta+\left(e^{P} Q^{2}+e^{-P}\right) d \delta^{2}\right]$.

Here, $\tau \in \mathbb{R}$ and $(\theta, \sigma, \delta)$ are coordinates on $T^{3}$. The functions $P, Q$ and $\lambda$ only depend on $\tau$ and $\theta$. The vacuum equations become

$$
\begin{aligned}
P_{\tau \tau}-e^{-2 \tau} P_{\theta \theta}-e^{2 P}\left(Q_{\tau}^{2}-e^{-2 \tau} Q_{\theta}^{2}\right) & =0 \\
Q_{\tau \tau}-e^{-2 \tau} Q_{\theta \theta}+2\left(P_{\tau} Q_{\tau}-e^{-2 \tau} P_{\theta} Q_{\theta}\right) & =0,
\end{aligned}
$$


and

$$
\begin{aligned}
& \lambda_{\tau}=P_{\tau}^{2}+e^{-2 \tau} P_{\theta}^{2}+e^{2 P}\left(Q_{\tau}^{2}+e^{-2 \tau} Q_{\theta}^{2}\right) \\
& \lambda_{\theta}=2\left(P_{\theta} P_{\tau}+e^{2 P} Q_{\theta} Q_{\tau}\right) .
\end{aligned}
$$

Obviously, the equations for $P$ and $Q$ are independent of $\lambda$, excepting the condition on $P$ and $Q$ implied by (5). The algorithm for producing a solution to (2)-(5) is thus to specify initial data to (2)-(3) such that the integral of the right hand side of (5) is zero, solve (2)-(3) and then find $\lambda$ by integrating (4)-(5). In the above parameterization, the singularity corresponds to $\tau \rightarrow \infty$, and by the above observations, it is clear that the main mathematical problem one is confronted with when studying the singularity in metrics of the form (1), is to analyze the asymptotics of solutions to (2)-(3) as $\tau \rightarrow \infty$. It is of interest to note that there is a special solution to the equations given by $P=\tau, Q=0$ and $\lambda=\tau$. The corresponding Lorentz metric has a curvature tensor which is identically zero. This is one of facts that make the analysis of Gowdy spacetimes interesting; there are special solutions that have the undesired property of having singularities that are not curvature singularities.

The equations (2)-(3) are wave map equations. In fact, let

$$
g_{0}=-e^{-2 \tau} d \tau^{2}+d \theta^{2}+e^{-2 \tau} d \chi^{2}
$$

be a Lorentz metric on $\mathbb{R} \times T^{2}$ and let

$$
g_{R}=d P^{2}+e^{2 P} d Q^{2}
$$

be a Riemannian metric on $\mathbb{R}^{2}$. Then (2)-(3) are the wave map equations for a map from $\left(\mathbb{R} \times T^{2}, g_{0}\right)$ to $\left(\mathbb{R}^{2}, g_{R}\right)$ which is independent of the $\chi$-coordinate. Note that $\left(\mathbb{R}^{2}, g_{R}\right)$ is isometric to the upper half plane $H=\left\{(x, y) \in \mathbb{R}^{2}: y>0\right\}$ with metric

$$
g_{H}=\frac{d x^{2}+d y^{2}}{y^{2}}
$$

under the map

$$
\phi_{R H}(Q, P)=\left(Q, e^{-P}\right) .
$$

Thus the target space is hyperbolic space. Note that due to the wave map structure, isometries of hyperbolic space take solutions to solutions. One particular isometry which will be of great use is the inversion, defined by

$$
\operatorname{Inv}(Q, P)=\left[\frac{Q}{Q^{2}+e^{-2 P}}, P+\ln \left(Q^{2}+e^{-2 P}\right)\right] .
$$

The reason for the name is that it corresponds to an inversion in the unit circle with center at the origin in the upper half plane model. Let us introduce the potential and kinetic energy densities, defined respectively by

$$
\begin{aligned}
& \mathcal{P}(\tau, \theta)=e^{-2 \tau}\left(P_{\theta}^{2}+e^{2 P} Q_{\theta}^{2}\right)(\tau, \theta) \\
& \mathcal{K}(\tau, \theta)=\left(P_{\tau}^{2}+e^{2 P} Q_{\tau}^{2}\right)(\tau, \theta) .
\end{aligned}
$$

In the analysis of Gowdy spacetimes, the existence of expansions for the solutions close to the singularity in certain situations is the key starting point. The idea of 
finding such expansions started with the paper [8] by Grubišić and Moncrief. In our setting, the natural expansions are

$$
\begin{aligned}
& P(\tau, \theta)=v(\theta) \tau+\phi(\theta)+u(\tau, \theta) \\
& Q(\tau, \theta)=q(\theta)+e^{-2 v(\theta) \tau}[\psi(\theta)+w(\tau, \theta)]
\end{aligned}
$$

where $w, u \rightarrow 0$ as $\tau \rightarrow \infty$ and $0<v(\theta)<1$. This should be compared with (5) and (6) of [10], where $-Z=P, X=Q$ and $t=e^{-\tau}$. Note that if we have a solution with the asymptotics (12)-(13) and $v(\theta)>0$, then $Q(\tau, \theta)$ converges to a finite limit and $P(\tau, \theta)$ tends to infinity as $\tau \rightarrow \infty$. Applying $\phi_{R H}$, we see that for a fixed $\theta$ the solution roughly speaking goes to the boundary along a geodesic in the upper half plane model. A heuristic argument motivating the condition on the velocity can be found in [2]. In the non-generic case $Q=0$, one can prove that (12) holds without any condition on $v$. This special case is called polarized Gowdy and has been studied in [9], which also considers the other topologies for Gowdy spacetimes. Due to the work of Kichenassamy and Rendall, [10], [12], one can specify smooth functions $v, \phi, q, \psi$, where $0<v<1$, and then obtain unique solutions to (2)-(3) with asymptotics of the form (12)-(13). For our applications it is of interest to note that if the $q$ one specifies is constant, the condition on $v$ can be relaxed to $v>0$.

According to our experience, the most important part of the expansions (12)-(13) is the function $v$. This is related to the intuition that the kinetic energy density is what controls everything else. Naively differentiating the expansions and computing $\mathcal{K}$, given in (11), one sees that this expression converges to $v^{2}$. In [16] we proved that the pointwise limit of $\mathcal{K}$ always exists. It therefore makes sense to make the following definition.

Definition 1.1. Consider a solution $x=(Q, P)$ to $(2)-(3)$. Then we define $v_{\infty}$ by

$$
v_{\infty}(\theta)=\left[\lim _{\tau \rightarrow \infty} \mathcal{K}(\tau, \theta)\right]^{1 / 2}
$$

If we wish to refer to the particular solution with respect to which it is defined, we shall write $v_{\infty}[x]$.

Define $\mathcal{D}_{\theta_{0}, \tau}=\left[\theta_{0}-e^{-\tau}, \theta_{0}+e^{-\tau}\right]$. One important result from [16] is the following.

Proposition 1.1. Consider a solution to (2)-(3) and let $\theta_{0} \in S^{1}$. Then

$$
\lim _{\tau \rightarrow \infty}\left\|\left|P_{\tau}(\tau, \cdot)\right|-v_{\infty}\left(\theta_{0}\right)\right\|_{C^{0}\left(\mathcal{D}_{\theta_{0}, \tau}, \mathbb{R}\right)}=0, \quad \lim _{\tau \rightarrow \infty}\left\|\left(e^{P} Q_{\tau}\right)(\tau, \cdot)\right\|_{C^{0}\left(\mathcal{D}_{\theta_{0}, \tau}, \mathbb{R}\right)}=0
$$

and

$$
\lim _{\tau \rightarrow \infty}\|\mathcal{P}(\tau, \cdot)\|_{C^{0}\left(\mathcal{D}_{\theta_{0}, \tau}, \mathbb{R}\right)}=0 .
$$

In particular, $P_{\tau}\left(\tau, \theta_{0}\right)$ converges to $v_{\infty}\left(\theta_{0}\right)$ or to $-v_{\infty}\left(\theta_{0}\right)$. If $P_{\tau}\left(\tau, \theta_{0}\right) \rightarrow-v_{\infty}\left(\theta_{0}\right)$, then $\left(Q_{1}, P_{1}\right)=\operatorname{Inv}(Q, P)$ has the property that $P_{1 \tau}\left(\tau, \theta_{0}\right) \rightarrow v_{\infty}\left(\theta_{0}\right)$. Furthermore, if $v_{\infty}\left(\theta_{0}\right)>0$, then $Q_{1}\left(\tau, \theta_{0}\right)$ converges to 0 .

Remark. In the above statement, $C^{0}$ is the space of continuous functions and $S^{1}$ is the unit circle, i.e. the interval $[0,2 \pi]$ with the endpoints identified.

Let us give an idea of the proof. The proof is based on a study of the quantity

$$
F_{\theta_{0}}(\tau)=\frac{1}{2} \sum_{ \pm}\left\|\left[\left(P_{\tau} \pm e^{-\tau} P_{\theta}\right)^{2}+e^{2 P}\left(Q_{\tau} \pm e^{-\tau} Q_{\theta}\right)^{2}\right](\tau, \cdot)\right\|_{C^{0}\left(\mathcal{D}_{\theta_{0}, \tau}, \mathbb{R}\right)} .
$$


Note that it dominates $\mathcal{P}+\mathcal{K}$ in $\mathcal{D}_{\theta_{0}, \tau}$. It turns out that this quantity is monotonically decaying, so that it converges, since it is bounded from below. Furthermore, by looking at the estimates in detail, there is strict decay if $\mathcal{P}$ is non-zero. Intuitively, one thus expects $\mathcal{P}$ to converge to zero in $\mathcal{D}_{\theta_{0}, \tau}$. In order to turn the intuition into a proof, one needs to be able to estimate the variation of $\mathcal{P}$ and $\mathcal{K}$, which is the first step. One then observes that one only needs to focus on a small neighbourhood of the characteristics, due to how information propagates. These things can be combined to prove that $\mathcal{P}$ has to converge to zero along the characteristics ending at $\theta_{0}$. Due to the concentration of information along characteristics, one can then prove that the liminf of $\mathcal{K}$ along characteristics dominates $F_{\theta_{0}}$. This can in its turn be used to prove that $\mathcal{P}$ converges to 0 in $\mathcal{D}_{\theta_{0}, \tau}$. One can then conclude that the variation of $\mathcal{P}$ and $\mathcal{K}$ inside $\mathcal{D}_{\theta_{0}, \tau}$ converges to zero and that $\mathcal{K}$ has to converge to something. Combining these observations with a study of $P_{\tau}+e^{-\tau} P_{\theta}$ along the characteristic $\left(\tau, \theta_{0}+e^{-\tau}\right)$ and the equations, one obtains the conclusions of the proposition.

The importance of the asymptotic velocity comes from the fact that if $v_{\infty}\left(\theta_{0}\right) \neq 1$, then the curvature blows up along any causal curve ending on $\theta_{0}$. Let us be more precise. Given an inextendible causal curve $\gamma$, the $\theta$ coordinate converges as the curve tends to the singularity. Let us call this coordinate $\theta_{0}[\gamma]$. In [16], we proved that if $v_{\infty}\left(\theta_{0}[\gamma]\right) \neq 1$, then

$$
\left(R_{\alpha \beta \mu \nu} R^{\alpha \beta \mu \nu}\right)[\gamma(s)] \rightarrow \infty
$$

as $s$ tends towards the parameter value corresponding to the singularity. Let $Y$ be the set of smooth initial data to (2)-(3). This set is in one-to-one correspondence with solutions to (2)-(3), and for the sake of definiteness, we shall take it to be understood that given $\xi \in Y$, we obtain a solution $x$ to (2)-(3) by specifying that the initial data of $x$ at $\tau=0$ be $\xi$. Note that an element of $Y$ does not necessarily correspond to a solution of the Einstein vacuum equations. In order to get a solution, the integral of the right hand side of (5) has to be zero. This is however the only condition. Let us denote the set of smooth initial data such that the integral of the right hand side is zero by $Y_{c}$. We shall consider $Y$ and $Y_{c}$ to be topological spaces by endowing them with the $C^{\infty}$ topology. Let $\mathcal{G}$ be the set of smooth initial data such that the corresponding asymptotic velocity is different from 1 on a dense subset of the singularity. Define $\mathcal{G}_{c}=\mathcal{G} \cap Y_{c}$. We wish to prove that $\mathcal{G}_{c}$, considered as a subset of $Y_{c}$, is a dense $G_{\delta}$ set. Recall that a set that can be written as a countable intersection of open sets is called a $G_{\delta}$ set. Consider a solution to (2)-(3). If the initial data are such that $v_{\infty} \neq 1$ on a dense subset of the singularity, there is not much more to be said. What remains to be analyzed is thus the situation in which $v_{\infty}=1$ on an open subset of the singularity.

Theorem 1.1. Consider a solution $(Q, P)$ to (2)-(3). Assume that $v_{\infty}=1$ in an open neighbourhood $J$ of $\theta_{0}$ and that $P_{\tau}\left(\tau, \theta_{0}\right) \rightarrow 1$ as $\tau \rightarrow \infty$. Then there is an open $I \subseteq J$ containing $\theta_{0}, \phi, r \in C^{\infty}(I, \mathbb{R})$ and constants $q_{0}$ and $C_{k}$ such that for 
all $k \geq 0$ and $\tau \geq 0$,

$$
\begin{aligned}
\left\|P_{\tau}(\tau, \cdot)-1\right\|_{C^{k}(I, \mathbb{R})} & \leq C_{k} e^{-2 \tau}, \\
\|P(\tau, \cdot)-\tau-\phi\|_{C^{k}(I, \mathbb{R})} & \leq C_{k} e^{-2 \tau}, \\
\left\|\left[e^{2 p} Q_{\tau}-r\right](\tau, \cdot)\right\|_{C^{k}(I, \mathbb{R})} & \leq C_{k} e^{-2 \tau}, \\
\left\|\left[e^{2 p}\left(Q-q_{0}\right)+\frac{r}{2}\right](\tau, \cdot)\right\|_{C^{k}(I, \mathbb{R})} & \leq C_{k} e^{-2 \tau},
\end{aligned}
$$

where $p(\tau, \theta)=\tau+\phi(\theta)$.

Remark. In [16] we have proven a similar result in which the asymptotic velocity is allowed to be non-constant and pass through 1 . To prove such a result one has to make some additional assumptions and it requires more of an effort. For the purposes of the present paper the above result is however sufficient.

The proof will be given at the end of section 3 .

In order to prove the desired result, it turns out that all one needs to do is to prove the following. Consider a solution with smooth expansions of the form (14)-(17) in some open interval $I$. Then there is a sequence of solutions converging to it, in the $C^{\infty}$ topology on initial data, which have $v_{\infty} \neq 1$ on some point of $I$. To obtain such a result, one uses the Fuchsian techniques developed in [10] and [12].

Theorem 1.2. With notation as above, $\mathcal{G}_{c}$ is dense in $Y_{c}$. Furthermore, $\mathcal{G}_{c}$ can be written as a countable intersection of open and dense subsets of $Y_{c}$.

Remark. By definition, a solution $(Q, P)$ corresponding to initial data in $\mathcal{G}_{c}$ has the property that the asymptotic velocity is different from 1 on a dense subset of the singularity. By the discussion following Proposition 1.1, we conclude that the corresponding Gowdy metric has the property that it exhibits curvature blow up on a dense subset of the singularity. Finally, note that the intersection of a countable number of open and dense sets in a complete metric space is dense, so that all one needs to prove is the second statement of the theorem.

The above theorem follows from Lemma 4.1 below. As was mentioned in the abstract, we have recently obtained a stronger result. It is contained in [16] and [17]. Since this paper uses the results of [16], it is natural to ask the question what purpose it serves. To start with, the results presented here preceded the results of [16] and [17]. Secondly, [16] is roughly 60 pages and [17] is roughly 50 pages long. For the results presented here, we only need two sections of in total 11 pages from [16]. In other words, the present proof is considerably shorter. On the other hand, as opposed to the present paper, [16] and [17] do not use the results of [10] and [12]. Finally, we would like to take this opportunity to draw the attention of the reader to the related work in [4] and [6]. In [4], the authors prove, among other things, that the asymptotic velocity makes sense on an open and dense subset of the singularity and that there are asymptotic expansions in a neighbourhood of these points. Furthermore, the authors study the cases where the velocity equals 1 or 0 . As was mentioned in the abstract, according to [6], the results of this paper can be combined with those of [6] in order to prove strong cosmic censorship for $T^{3}$-Gowdy spacetimes. 


\section{NotATion}

Let $(Q, P)$ be a solution to (2)-(3). Define

$$
\mathcal{A}_{k, \pm}=\frac{1}{2} e^{\tau}\left[\left(\partial_{\tau} \partial_{\theta}^{k} P \pm e^{-\tau} \partial_{\theta}^{k+1} P\right)^{2}+e^{2 P}\left(\partial_{\tau} \partial_{\theta}^{k} Q \pm e^{-\tau} \partial_{\theta}^{k+1} Q\right)^{2}\right] .
$$

In order to obtain estimates for the higher derivatives, let us compute

$$
\left(\partial_{\tau} \mp e^{-\tau} \partial_{\theta}\right) \mathcal{A}_{k, \pm}=I_{1, k, \pm}+I_{2, k, \pm}
$$

where

$$
\begin{aligned}
I_{1, k, \pm}= & \frac{1}{2} e^{\tau}\left\{\left(\partial_{\tau} \partial_{\theta}^{k} P\right)^{2}-e^{-2 \tau}\left(\partial_{\theta}^{k+1} P\right)^{2}+e^{2 P}\left[\left(\partial_{\tau} \partial_{\theta}^{k} Q\right)^{2}-e^{-2 \tau}\left(\partial_{\theta}^{k+1} Q\right)^{2}\right]\right\} \\
& -e^{2 P+\tau}\left(P_{\tau} \pm e^{-\tau} P_{\theta}\right)\left[\left(\partial_{\tau} \partial_{\theta}^{k} Q\right)^{2}-e^{-2 \tau}\left(\partial_{\theta}^{k+1} Q\right)^{2}\right] \\
& +e^{2 P+\tau}\left(Q_{\tau} \pm e^{-\tau} Q_{\theta}\right)\left[\left(\partial_{\tau} \partial_{\theta}^{k} P \pm e^{-\tau} \partial_{\theta}^{k+1} P\right)\left(\partial_{\tau} \partial_{\theta}^{k} Q \mp e^{-\tau} \partial_{\theta}^{k+1} Q\right)\right. \\
& \left.-\left(\partial_{\tau} \partial_{\theta}^{k} P \mp e^{-\tau} \partial_{\theta}^{k+1} P\right)\left(\partial_{\tau} \partial_{\theta}^{k} Q \pm e^{-\tau} \partial_{\theta}^{k+1} Q\right)\right]
\end{aligned}
$$

and

$$
\begin{aligned}
I_{2, k, \pm}= & e^{\tau}\left\{\partial_{\theta}^{k}\left[e^{2 P}\left(Q_{\tau}^{2}-e^{-2 \tau} Q_{\theta}^{2}\right)\right]-2 e^{2 P}\left(Q_{\tau} \partial_{\theta}^{k} \partial_{\tau} Q-e^{-2 \tau} Q_{\theta} \partial_{\theta}^{k+1} Q\right)\right\} \\
& \cdot\left(\partial_{\tau} \partial_{\theta}^{k} P \pm e^{-\tau} \partial_{\theta}^{k+1} P\right)+e^{2 P+\tau} \sum_{l=1}^{k-1}\left(\begin{array}{c}
k \\
l
\end{array}\right)\left[-2 \partial_{\theta}^{k-l} \partial_{\tau} P \partial_{\theta}^{l} \partial_{\tau} Q\right. \\
& \left.+2 e^{-2 \tau} \partial_{\theta}^{k-l+1} P \partial_{\theta}^{l+1} Q\right]\left(\partial_{\tau} \partial_{\theta}^{k} Q \pm e^{-\tau} \partial_{\theta}^{k+1} Q\right) .
\end{aligned}
$$

If $k \leq 1$, the sum is taken to be zero. If $I=[a, b]$ is a subinterval of $\mathbb{R}$, let

$$
\mathcal{D}_{I, \tau}=\left[a-e^{-\tau}, b+e^{-\tau}\right] .
$$

The definition if $I$ is an open interval is similar. If $\mathcal{D}_{I, \tau}$ has a length which equals or exceeds that of the circle, we shall interpret it as the whole circle.

2.1. Equations in the disc model. It is sometimes convenient to consider the equations in the disc model, where they take the form

$$
\partial_{\tau}\left(\frac{z_{\tau}}{\left(1-|z|^{2}\right)^{2}}\right)-e^{-2 \tau} \partial_{\theta}\left(\frac{z_{\theta}}{\left(1-|z|^{2}\right)^{2}}\right)=\frac{2 z}{\left(1-|z|^{2}\right)^{3}}\left[\left|z_{\tau}\right|^{2}-e^{-2 \tau}\left|z_{\theta}\right|^{2}\right]
$$

The easiest way to see this is to use the action

$$
\iint\left[P_{\tau}^{2}+e^{2 P} Q_{\tau}^{2}-e^{-2 \tau}\left(P_{\theta}^{2}+e^{2 P} Q_{\theta}^{2}\right)\right] d \theta d \tau
$$

in order to derive the Gowdy equations. It translates into

$$
\iint \frac{4\left(\left|z_{\tau}\right|^{2}-e^{-2 \tau}\left|z_{\theta}\right|^{2}\right)}{\left(1-|z|^{2}\right)^{2}} d \theta d \tau
$$

in the disc model. Note that the isometry from $\left(\mathbb{R}^{2}, g_{R}\right)$ to the disc model defined by

$$
\phi_{R D}(Q, P)=\frac{Q+i\left(e^{-P}-1\right)}{Q+i\left(e^{-P}+1\right)}
$$

defines an injective and surjective map of solutions to (2)-(3) to solutions of (21). We shall also use $(\rho, \phi)$ as variables. They are defined by

$$
z=|z| e^{i \phi}, \quad \rho=\ln \frac{1+|z|}{1-|z|} .
$$


Note that $\rho$ is the hyperbolic distance from the origin of the disc to the solution. In the end we are only interested in the absolute value of derivatives of $\phi$, and since

$$
\phi_{\tau}^{2}=\left|\partial_{\tau}\left(\frac{z}{|z|}\right)\right|^{2}
$$

it is clear that these make sense as long as $|z|>0$. It is useful to keep in mind that

$$
\rho_{\tau}^{2}+\sinh ^{2} \rho \phi_{\tau}^{2}=\frac{4\left|z_{\tau}\right|^{2}}{\left(1-|z|^{2}\right)^{2}}
$$

and similarly for the $\theta$-derivatives. It will be convenient to have the inverse of $(22)$,

$$
(Q, P)=\left[-\frac{2 \operatorname{Im} z}{|1-z|^{2}},-\ln \left(1-|z|^{2}\right)+2 \ln |1-z|\right] .
$$

Using the definition of $\rho$, this yields

$$
P=\rho-2 \ln (1+|z|)+2 \ln |1-z| .
$$

\section{Asymptotic expansions}

The purpose of this section is to prove Theorem 1.1. The essential assumptions of the theorem are that $v_{\infty}=1$ in an open set. In other words, we only make assumptions concerning the pointwise limit of $\mathcal{K}$. The conclusions, on the other hand, contain statements concerning uniform limits of an arbitrary number of derivatives. Let us point out that the potential energy $\mathcal{P}$ converges to zero everywhere, but there are examples where it does not converge to zero uniformly. The step from pointwise to uniform convergence is therefore not necessarily a triviality. Lemma 3.1 serves the purpose of taking the step from pointwise to uniform convergence for the first derivatives. Furthermore, the result that $P-\tau$ is bounded is very convenient to have since factors of the form $e^{P-\tau}$ appear frequently, and it is of interest to estimate them from above and from below. Finally, due to the fact that $Q_{\theta}$ converges to zero uniformly, which is a consequence of the lemma below, if $\partial_{\tau} \partial_{\theta}^{k} Q$ decays exponentially for some $k \geq 1$, then $\partial_{\theta}^{k} Q$ decays exponentially (since we know that if $\partial_{\theta}^{k} Q$ converges, it has to converge to zero). This will also be very useful information in the derivation of the estimates for the higher derivatives.

Lemma 3.1. Consider a solution $x=(Q, P)$ to (2)-(3). Assume that $v_{\infty}=1$ in an open neighbourhood $J$ of $\theta_{0}$ and that $P_{\tau}\left(\tau, \theta_{0}\right) \rightarrow 1$ as $\tau \rightarrow \infty$. Then there is an open $I \subseteq J$ containing $\theta_{0}$ and a constant $C$ such that for $\tau \geq 1$

$$
\begin{aligned}
\|P(\tau, \cdot)-\tau\|_{C^{0}\left(\mathcal{D}_{I, \tau}, \mathbb{R}\right)} & \leq C \\
\left\|\left[\left(P_{\tau}-1\right)^{2}+e^{-2 \tau} P_{\theta}^{2}+e^{2 P}\left(Q_{\tau}^{2}+e^{-2 \tau} Q_{\theta}^{2}\right)\right](\tau, \cdot)\right\|_{C^{0}\left(\mathcal{D}_{I, \tau}, \mathbb{R}\right)} & \leq C \tau^{-2} .
\end{aligned}
$$

Remark. As a consequence, $Q$ converges to a constant in $C^{1}(I, \mathbb{R})$.

Proof. We can consider the solution as being defined for $\theta \in \mathbb{R}$ by making it $2 \pi$-periodic in $\theta$. The relations

$$
P_{1}=\tau-P, \quad Q_{1 \tau}=-e^{2(P-\tau)} Q_{\theta}, \quad Q_{1 \theta}=-e^{2 P} Q_{\tau}
$$

define a new solution to (2)-(3) with $\theta \in \mathbb{R}$. The definition is up to a constant, but the value of the constant is of no importance. Note that the resulting solution need not be $2 \pi$-periodic. This is the so called Gowdy to Ernst transformation, which has been used for example in [13]. Let $x_{1}=\left(Q_{1}, P_{1}\right)$. By construction and Proposition 
$1.1, v_{\infty}\left[x_{1}\right]\left(\theta_{0}\right)=0$. Let $z=\phi_{R D} \circ x_{1}$, where $\phi_{R D}$ is defined in (22). Then $z$ is a solution to $(21)$ with $\theta \in \mathbb{R}$. Since $\phi_{R D}$ is an isometry, we conclude that $\mathcal{K}\left(\tau, \theta_{0}\right)$ converges to zero, where

$$
\mathcal{K}=\frac{4\left|z_{\tau}\right|^{2}}{\left(1-|z|^{2}\right)^{2}}
$$

Due to (24), we conclude that $\rho\left(\tau, \theta_{0}\right) / \tau$ converges to zero, where $\rho$ is defined as in (23). Due to Lemma 7 of [15], we conclude that there is an open neighbourhood $I$ of $\theta_{0}$ contained in $J$, a function $v \in C^{0}\left(I, \mathbb{R}^{2}\right)$ and a $C$ such that for $\tau \geq 1$,

$$
\begin{gathered}
\left\|\frac{1}{\tau} \frac{z(\tau, \cdot)}{|z(\tau, \cdot)|} \rho(\tau, \cdot)-v\right\|_{C^{0}\left(I, \mathbb{R}^{2}\right)}+\left\|\frac{2 z_{\tau}(\tau, \cdot)}{1-|z(\tau, \cdot)|^{2}}-v\right\|_{C^{0}\left(I, \mathbb{R}^{2}\right)} \\
+e^{-\tau}\left\|\frac{2 z_{\theta}(\tau, \cdot)}{1-|z(\tau, \cdot)|^{2}}\right\|_{C^{0}\left(I, \mathbb{R}^{2}\right)} \leq C \tau^{-1} .
\end{gathered}
$$

Since $v\left(\theta_{0}\right)=0$ and $v$ is continuous, we can assume that $|v|<1 / 2$ in $I$. This implies that $v_{\infty}\left[x_{1}\right](\theta)<1 / 2$. Since $v_{\infty}[x]=1$ in $I$ and since we have Proposition 1.1 , the only possibilities for $v_{\infty}\left[x_{1}\right]$ in $I$ are 0 or 2 , so that $v_{\infty}\left[x_{1}\right]=0$ in $I$. Thus $v=0$ in $I$. In particular, this means that $\rho(\tau, \theta)$ is bounded by a constant for $\tau \geq 1$ and $\theta \in I$. Due to (26), this leads to the conclusion that $P_{1}$ is bounded in the same set, which leads to the first conclusion of the lemma, with $\mathcal{D}_{I, \tau}$ replaced by $I$, due to the definition of $P_{1}$. Furthermore, we get the conclusion that the kinetic and potential energy densities of $x_{1}$ decay like $\tau^{-2}$. Compute

$\left(P_{\tau}-1\right)^{2}+e^{-2 \tau} P_{\theta}^{2}+e^{2 P}\left(Q_{\tau}^{2}+e^{-2 \tau} Q_{\theta}^{2}\right)=P_{1 \tau}^{2}+e^{-2 \tau} P_{1 \theta}^{2}+e^{2 P_{1}}\left(Q_{1 \tau}^{2}+e^{-2 \tau} Q_{1 \theta}^{2}\right)$.

Since the right hand side is $O\left(\tau^{-2}\right)$, the second conclusion of the lemma follows. In reality, we only get the conclusions with $\mathcal{D}_{I, \tau}$ replaced by $I$, but this can be remedied by making $I$ smaller.

We need to improve the above estimates before we can use them to obtain estimates for the higher derivatives. In particular, it is important to go from $\tau^{-1}$ decay to exponential decay. It is also useful to have a first estimate for the second derivatives.

Lemma 3.2. Consider a solution $x=(Q, P)$ to (2)-(3). Assume that $v_{\infty}=1$ in an open neighbourhood $J$ of $\theta_{0}$ and that $P_{\tau}\left(\tau, \theta_{0}\right) \rightarrow 1$ as $\tau \rightarrow \infty$. Then there is an open neighbourhood $I$ of $\theta_{0}$, a polynomial $\Pi$ and a function $r \in C^{0}(I, \mathbb{R})$ such that for $\tau \geq 0$

$$
\begin{aligned}
\|P(\tau, \cdot)-\tau-r\|_{C^{0}(I, \mathbb{R})}+\left\|P_{\tau}(\tau, \cdot)-1\right\|_{C^{0}\left(\mathcal{D}_{I, \tau}, \mathbb{R}\right)} & \leq \Pi e^{-\tau}, \\
\left\|\left[e^{-2 \tau} P_{\theta}^{2}+e^{2 P}\left(Q_{\tau}^{2}+e^{-2 \tau} Q_{\theta}^{2}\right)\right](\tau, \cdot)\right\|_{C^{0}\left(\mathcal{D}_{I, \tau}, \mathbb{R}\right)} & \leq \Pi e^{-2 \tau} \\
\left\|\left[P_{\tau \theta}^{2}+e^{-2 \tau} P_{\theta \theta}^{2}+e^{2 P}\left(Q_{\tau \theta}^{2}+e^{-2 \tau} Q_{\theta \theta}^{2}\right)\right](\tau, \cdot)\right\|_{C^{0}\left(\mathcal{D}_{I, \tau}, \mathbb{R}\right)} & \leq \Pi .
\end{aligned}
$$

Proof. Note that the conditions of Lemma 3.1 are satisfied, so that there is an open set $I \subseteq J$ containing $\theta_{0}$ in which the conclusions of that lemma hold. By (18), we have

$$
\begin{aligned}
\left(\partial_{\tau} \mp e^{-\tau} \partial_{\theta}\right) \mathcal{A}_{1, \pm}= & \frac{1}{2} e^{\tau}\left[P_{\tau \theta}^{2}-e^{-2 \tau} P_{\theta \theta}^{2}+e^{2 P}\left(Q_{\tau \theta}^{2}-e^{-2 \tau} Q_{\theta \theta}^{2}\right)\right] \\
& +2 e^{\tau} P_{\theta} e^{2 P}\left(Q_{\tau}^{2}-e^{-2 \tau} Q_{\theta}^{2}\right)\left(P_{\tau \theta} \pm e^{-\tau} P_{\theta \theta}\right) \\
& -e^{2 P+\tau}\left(P_{\tau} \pm e^{-\tau} P_{\theta}\right)\left[Q_{\tau \theta}^{2}-e^{-2 \tau} Q_{\theta \theta}^{2}\right] \\
& +e^{2 P+\tau}\left(Q_{\tau} \pm e^{-\tau} Q_{\theta}\right)\left[\left(P_{\tau \theta} \pm e^{-\tau} P_{\theta \theta}\right)\left(Q_{\tau \theta} \mp e^{-\tau} Q_{\theta \theta}\right)\right. \\
& \left.-\left(P_{\tau \theta} \mp e^{-\tau} P_{\theta \theta}\right)\left(Q_{\tau \theta} \pm e^{-\tau} Q_{\theta \theta}\right)\right] .
\end{aligned}
$$


Define

$$
\mathcal{A}_{1, \pm}^{c}=\mathcal{A}_{1, \pm}+\frac{1}{2}\left(1+\tau^{2}\right)^{-1} e^{\tau} P_{\theta}^{2}
$$

Consider (28). By the conclusions of Lemma 3.1, we have

$$
\left(\partial_{\tau} \mp e^{-\tau} \partial_{\theta}\right) \mathcal{A}_{1, \pm} \leq \frac{1}{2}\left(\mathcal{A}_{1,+}+\mathcal{A}_{1,-}\right)+C \tau^{-1}\left(\mathcal{A}_{1,+}^{c}+\mathcal{A}_{1,-}^{c}\right)
$$

in $\mathcal{D}_{I, \tau}$ for $\tau \geq 1$. To obtain this inequality, we used the inequality $a b \leq\left(a^{2}+b^{2}\right) / 2$ in the term appearing on the second row, and replaced $P_{\tau}$ with 1 in the third row. Similarly, we can estimate

$$
\left(\partial_{\tau} \mp e^{-\tau} \partial_{\theta}\right)\left[\frac{1}{2}\left(1+\tau^{2}\right)^{-1} e^{\tau} P_{\theta}^{2}\right] \leq \frac{1}{2}\left(1+\tau^{2}\right)^{-1} e^{\tau} P_{\theta}^{2}+C \tau^{-1}\left(\mathcal{A}_{1,+}^{c}+\mathcal{A}_{1,-}^{c}\right) .
$$

Adding up, we get

$$
\left(\partial_{\tau} \mp e^{-\tau} \partial_{\theta}\right) \mathcal{A}_{1, \pm}^{c} \leq \frac{1}{2}\left(1+C \tau^{-1}\right)\left(\mathcal{A}_{1,+}^{c}+\mathcal{A}_{1,-}^{c}\right) .
$$

Define

$$
F_{I, 1}^{c}(\tau)=\sum_{ \pm}\left\|\mathcal{A}_{1, \pm}^{c}(\tau, \cdot)\right\|_{C^{0}\left(\mathcal{D}_{I, \tau}, \mathbb{R}\right)} \cdot
$$

Let $\theta \in \mathcal{D}_{I, \tau}$ and estimate

$$
\begin{aligned}
\mathcal{A}_{1, \pm}^{c}(\tau, \theta)= & \mathcal{A}_{1, \pm}^{c}\left(\tau_{0}, \theta \pm e^{-\tau_{0}} \mp e^{-\tau}\right) \\
& +\int_{\tau_{0}}^{\tau}\left[\left(\partial_{\tau} \mp e^{-s} \partial_{\theta}\right) \mathcal{A}_{1, \pm}^{c}\right]\left(s, \theta \pm e^{-s} \mp e^{-\tau}\right) d s \\
\leq & \left\|\mathcal{A}_{1, \pm}^{c}\right\|_{C^{0}\left(\mathcal{D}_{I, \tau_{0}}, \mathbb{R}\right)}+\int_{\tau_{0}}^{\tau} \frac{1}{2}\left(1+C s^{-1}\right) F_{I, 1}^{c}(s) d s .
\end{aligned}
$$

Taking the supremum over $\theta$ and adding the two estimates, we get

$$
F_{I, 1}^{c}(\tau) \leq F_{I, 1}^{c}\left(\tau_{0}\right)+\int_{\tau_{0}}^{\tau}\left(1+C s^{-1}\right) F_{I, 1}^{c}(s) d s .
$$

By Grönwall's Lemma, we obtain

(29) $\left\|\left[P_{\tau \theta}^{2}+e^{-2 \tau} P_{\theta \theta}^{2}+e^{2 P}\left(Q_{\tau \theta}^{2}+e^{-2 \tau} Q_{\theta \theta}^{2}\right)+\left(1+\tau^{2}\right)^{-1} P_{\theta}^{2}\right](\tau, \cdot)\right\|_{C^{0}\left(\mathcal{D}_{I, \tau}, \mathbb{R}\right)} \leq \Pi(\tau)$

for $\tau \geq 0$, where, here and below, $\Pi$ denotes some polynomial. This yields the last conclusion of the lemma. Compute

$$
\partial_{\tau}\left(e^{2 P} Q_{\tau}\right)=\partial_{\theta}\left(e^{2 P-2 \tau} Q_{\theta}\right)=2 P_{\theta} e^{2 P-2 \tau} Q_{\theta}+e^{2 P-2 \tau} Q_{\theta \theta}
$$

By (29) and Lemma 3.1, we conclude that the absolute value of the right hand side does not grow faster than polynomially. Consequently, $e^{P} Q_{\tau}$ decays as $\Pi e^{-\tau}$ in $I$. Since $e^{-\tau} \partial_{\theta}\left(e^{P} Q_{\tau}\right)$ satisfies the same sort of decay on $\mathcal{D}_{I, \tau}$, we can replace $I$ with $\mathcal{D}_{I, \tau}$ (here we use the bound $\left|e^{P} Q_{\tau}\right| \leq C$ outside of $I$, which follows from Proposition 1.1 applied to the endpoints). Since $Q_{\tau \theta}$ decays as $\Pi e^{-\tau}$ and $Q_{\theta}$ converges to zero due to Lemma 3.1, we conclude that $e^{P-\tau} Q_{\theta}$ decays as $\Pi e^{-\tau}$ on $I$. The argument for going from $I$ to $\mathcal{D}_{I, \tau}$ is similar to the previous case. This, together with (29), yields the second to last conclusion of the lemma. Combining the information we have obtained with (2), we conclude that (27) holds.

Let us turn to the proof of Proposition 3.1. The proof is by induction. We assume that (33) holds for $k$ and wish to prove that it holds for $k+1$. In each induction step, it is necessary to improve the estimates for $e^{P} \partial_{\theta}^{k} Q$ and $e^{P} \partial_{\theta}^{k-1} \partial_{\tau} Q$. In order not to make the proof of Proposition 3.1 too long, we prove these steps separately 
in Lemma 3.3 and 3.3. In the following lemmas, we shall assume that we have an open set $I$ in which the conclusions of Lemma 3.2 hold. Note in particular that $P-\tau$ is bounded in $\mathcal{D}_{I, \tau}$ for $\tau \geq 0$.

Lemma 3.3. With the same notation and assumptions as in Lemma 3.2, assume that

$$
\left\|e^{2 P}\left(\partial_{\theta}^{k} \partial_{\tau} Q\right)^{2}+e^{2 P-2 \tau}\left(\partial_{\theta}^{k+1} Q\right)^{2}\right\|_{C^{0}\left(\mathcal{D}_{I, \tau}, \mathbb{R}\right)} \leq C,
$$

for some $k \geq 1$. Then

$$
\left\|e^{2 P-2 \tau}\left(\partial_{\theta}^{k} Q\right)^{2}\right\|_{C^{0}\left(\mathcal{D}_{I, \tau}, \mathbb{R}\right)} \leq C e^{-2 \tau}
$$

Remark. Note that if we replace the constant $C$ in the assumptions with a polynomial, we get the same conclusions if we take $C$ to be a polynomial.

Proof. Due to (27) and the assumptions of the lemma, we have

$$
\left|\partial_{\theta}^{k} \partial_{\tau} Q\right| \leq C e^{-\tau}
$$

Furthermore, by Lemma 3.2, we know that $Q_{\theta}$ converges to zero. We obtain the conclusion with $\mathcal{D}_{I, \tau}$ replaced by $I$. By the estimate we have on $e^{P} \partial_{\theta}^{k+1} Q$ we then obtain the desired conclusion.

Lemma 3.4. With the same notation and assumptions as in Lemma 3.2, assume that

$$
\left\|\left(\partial_{\theta}^{k} \partial_{\tau} P\right)^{2}+e^{-2 \tau}\left(\partial_{\theta}^{k+1} P\right)^{2}+e^{2 P}\left[\left(\partial_{\theta}^{k} \partial_{\tau} Q\right)^{2}+e^{-2 \tau}\left(\partial_{\theta}^{k+1} Q\right)^{2}\right]\right\|_{C^{0}\left(\mathcal{D}_{I, \tau}, \mathbb{R}\right)} \leq C_{k}
$$

for $k=0, \ldots, l$, where $l \geq 1$. Then

$$
\left\|e^{2 P}\left(\partial_{\theta}^{m} \partial_{\tau} Q\right)^{2}\right\|_{C^{0}\left(\mathcal{D}_{I, \tau}, \mathbb{R}\right)} \leq \Pi_{m}(\tau) e^{-2 \tau},
$$

for $m \leq l-1$, where $\Pi_{m}$ is a polynomial.

Remark. Note that if we replace $C_{k}$ in the conditions with a polynomial, we get the same conclusion.

Proof. Let us compute

$$
\begin{aligned}
\partial_{\tau}\left(e^{2 P} \partial_{\theta}^{m} \partial_{\tau} Q\right)= & e^{2 P-2 \tau} \partial_{\theta}^{m+2} Q-2 e^{2 P} \sum_{j=1}^{m}\left(\begin{array}{c}
m \\
j
\end{array}\right) \partial_{\theta}^{j} \partial_{\tau} P \partial_{\theta}^{m-j} \partial_{\tau} Q \\
& +2 e^{2 P-2 \tau} \sum_{j=0}^{m}\left(\begin{array}{c}
m \\
j
\end{array}\right) \partial_{\theta}^{j+1} P \partial_{\theta}^{m-j+1} Q .
\end{aligned}
$$

By Lemma 3.2 we know that the conclusion holds for $l=1$. Assume now that the statement of the lemma is true up to and including some $l \geq 1$, and assume that the assumptions of the lemma hold with $l$ replaced with $l+1$. Consider (32) for $m=l$. The first term is bounded by the assumptions. By the assumptions and the inductive assumptions, we conclude that the second term is at worst polynomial. Since $\partial_{\theta}^{j} \partial_{\tau} P$ is bounded for $j \leq l+1$, we conclude that $\partial_{\theta}^{j} P$ grows at worst polynomially for $j \leq l+1$. Combining this observation with Lemma 3.3, we conclude that the last term is exponentially decaying. The conclusion of the lemma follows with $\mathcal{D}_{I, \tau}$ replaced with $I$, a problem which can be dealt with as before. 
Proposition 3.1. With the same notation and assumptions as in Lemma 3.2, we have

(33) $\left\|\left(\partial_{\theta}^{k} \partial_{\tau} P\right)^{2}+e^{-2 \tau}\left(\partial_{\theta}^{k+1} P\right)^{2}+e^{2 P}\left[\left(\partial_{\theta}^{k} \partial_{\tau} Q\right)^{2}+e^{-2 \tau}\left(\partial_{\theta}^{k+1} Q\right)^{2}\right]\right\|_{C^{0}\left(\mathcal{D}_{I, \tau}, \mathbb{R}\right)} \leq C_{k}$ for all $k \geq 0$ and $\tau \geq 0$.

Proof. Consider (18), with $I_{1, k, \pm}$ and $I_{2, k, \pm}$ defined in (19) and (20). By Lemma 3.2

$$
I_{1, k, \pm} \leq\left(\frac{1}{2}+\Pi e^{-\tau}\right)\left(\mathcal{A}_{k,+}+\mathcal{A}_{k,-}\right)
$$

If it were only for this term, we would thus be done. To the extent that there are complications, they arise due to $I_{2, k, \pm}$. Define

$$
\hat{\mathcal{A}}_{k, \pm}=\mathcal{A}_{k, \pm}+\frac{1}{2} e^{\tau / 2}\left(\partial_{\theta}^{k} P\right)^{2} .
$$

Let us start by proving the lemma for $k=1$. We have

$$
I_{2,1, \pm}=2 e^{\tau} P_{\theta} e^{2 P}\left(Q_{\tau}^{2}-e^{-2 \tau} Q_{\theta}^{2}\right)\left(P_{\tau \theta} \pm e^{-\tau} P_{\theta \theta}\right) \leq \Pi e^{-7 \tau / 4}\left(\hat{\mathcal{A}}_{1,+}+\hat{\mathcal{A}}_{1,-}\right) .
$$

Since

$$
\left(\partial_{\tau} \mp e^{-\tau} \partial_{\theta}\right)\left[\frac{1}{2} e^{\tau / 2}\left(\partial_{\theta}^{k} P\right)^{2}\right] \leq \frac{1}{2} e^{\tau / 2}\left(\partial_{\theta}^{k} P\right)^{2}+C e^{-\tau / 4}\left(\hat{\mathcal{A}}_{k,+}+\hat{\mathcal{A}}_{k,-}\right),
$$

we get

$$
\left(\partial_{\tau} \mp e^{-\tau} \partial_{\theta}\right) \hat{\mathcal{A}}_{1, \pm} \leq\left(\frac{1}{2}+C e^{-\tau / 4}\right)\left(\hat{\mathcal{A}}_{1,+}+\hat{\mathcal{A}}_{1,-}\right)
$$

Thus

$$
\hat{F}_{I, 1}(\tau) \leq \hat{F}_{I, 1}\left(\tau_{0}\right)+\int_{\tau_{0}}^{\tau}\left[1+C e^{-s / 4}\right] \hat{F}_{I, 1}(s) d s
$$

where

$$
\hat{F}_{I, k}(\tau)=\sum_{ \pm} \sup _{\theta \in \mathcal{D}_{I, \tau}} \hat{\mathcal{A}}_{k, \pm}(\tau, \theta)
$$

By Grönwall's lemma, we obtain the conclusion for $k=1$. Assume inductively that we have the conclusion up to and including $k$. We wish to prove that the conclusion holds for $k+1$. By the inductive hypothesis

$$
\left\|e^{2 P}\left[\left(\partial_{\theta}^{l} \partial_{\tau} Q\right)^{2}+e^{-2 \tau}\left(\partial_{\theta}^{l+1} Q\right)^{2}\right)\right\|_{C^{0}\left(\mathcal{D}_{I, \tau}, \mathbb{R}\right)} \leq \Pi_{l}(\tau) e^{-2 \tau},
$$

for $l \leq k-1$ due to lemmas 3.3 and 3.4. By the inductive hypothesis, we also know that $\partial_{\theta}^{l} P$ does not grow faster than polynomially if $l \leq k$. Consider the two different terms of $I_{2, k+1, \pm}$. The first can be rewritten

$\sum_{i, j, l} a_{i j l} \partial_{\theta}^{i}\left(e^{2 P+\tau}\right)\left[\left(\partial_{\theta}^{j} \partial_{\tau} Q\right)\left(\partial_{\theta}^{l} \partial_{\tau} Q\right)-e^{-2 \tau}\left(\partial_{\theta}^{j+1} Q\right)\left(\partial_{\theta}^{l+1} Q\right)\right]\left(\partial_{\tau} \partial_{\theta}^{k+1} P \pm e^{-\tau} \partial_{\theta}^{k+2} P\right)$,

where $i+j+l=k+1$ and $j, l \leq k$. If $i \leq k$, no derivatives of $P$ of higher order than $k$ appear, and such terms can be bounded by polynomials. If $i=1+k$, there is a problem if all $k+1$ derivatives hit "the same" $P$. However, then (34) with $l=0$ and the fact that $e^{-\tau} \partial_{\theta}^{k+1} P$ is bounded yields the conclusion that the corresponding term can be bounded by

$$
\Pi(\tau) e^{-\tau / 2}\left(\mathcal{A}_{k+1,+}+\mathcal{A}_{k+1,-}\right)^{1 / 2} .
$$

In fact this sort of bound holds as long as $j, l \leq k-1$ (in fact we get a bound with $e^{-\tau / 2}$ replaced with $e^{-3 \tau / 2}$ if in addition $\left.i \leq k\right)$. Assume therefore that $j=k$. If 
$i=1$, we get a bound of the form (35) by the induction hypothesis and arguments already mentioned. If $l=1$, there are two cases. If $k \geq 2$, we get a bound of the form (35), but if $k=1$, we get the bound

$$
C e^{\tau / 2}\left(\mathcal{A}_{k+1,+}+\mathcal{A}_{k+1,-}\right)^{1 / 2} .
$$

Consider the second term in $I_{2, k+1, \pm}$. We need to estimate terms of the form

$$
e^{2 P+\tau}\left[-2 \partial_{\theta}^{k+1-l} \partial_{\tau} P \partial_{\theta}^{l} \partial_{\tau} Q+2 e^{-2 \tau} \partial_{\theta}^{k-l+2} P \partial_{\theta}^{l+1} Q\right]\left(\partial_{\tau} \partial_{\theta}^{k+1} Q \pm e^{-\tau} \partial_{\theta}^{k+2} Q\right),
$$

where $1 \leq l \leq k$. By the induction hypothesis, terms of this form can all be estimated by (36). Adding up the above observations, we get the conclusion that

$$
\begin{aligned}
& \left(\partial_{\tau} \mp e^{-\tau} \partial_{\theta}\right) \mathcal{A}_{k+1, \pm} \\
& \quad \leq\left(\frac{1}{2}+\Pi e^{-\tau}\right)\left(\mathcal{A}_{k+1,+}+\mathcal{A}_{k+1,-}\right)+C e^{\tau / 2}\left(\mathcal{A}_{k+1,+}+\mathcal{A}_{k+1,-}\right)^{1 / 2} .
\end{aligned}
$$

Thus

$$
F_{I, k+1}(\tau) \leq F_{I, k+1}\left(\tau_{0}\right)+\int_{\tau_{0}}^{\tau}\left[\left(1+\Pi e^{-s}\right) F_{I, k+1}(s)+C e^{s / 2} F_{I, k+1}^{1 / 2}(s)\right] d s .
$$

Unfortunately, this does not lead to exactly what we want. We only get a polynomial bound. However, combining the polynomial bound with Lemmas 3.3 and 3.4, we get better decay estimates which can be used to improve the estimates described in the proof of this lemma. In this way we get the desired conclusion.

Proof of Theorem 1.1. By the assumptions, we have the conclusions of Lemma 3.2 and Proposition 3.1. Below we shall only consider the behaviour for $\theta \in I$. Note that the conditions of Lemmas 3.3 and 3.4 are fulfilled and the consequences of this will be used freely below. Note also that $\partial_{\theta}^{k+1} P$ does not grow faster than linearly. Inserting these observations into (2), we get the conclusion that $\partial_{\theta}^{k} \partial_{\tau}^{2} P$ decays to zero as a polynomial times $e^{-2 \tau}$. Since we know that $P_{\tau}$ converges to 1 , we can integrate this inequality twice in order to obtain the conclusion that there is a smooth function $\phi$ and a polynomial $\Xi_{k}$ such that

$$
\left\|P_{\tau}(\tau, \cdot)-1\right\|_{C^{k}(I, \mathbb{R})}+\|P(\tau, \cdot)-\tau-\phi\|_{C^{k}(I, \mathbb{R})} \leq \Xi_{k}(\tau) e^{-2 \tau}
$$

Note that in particular, $\partial_{\theta}^{k} \partial_{\tau} P$ converges to zero exponentially and $\partial_{\theta}^{k} P$ is bounded if $k \geq 1$. Inserting this information into (32), we conclude that we can replace the polynomial $\Pi_{m}$ in (31) with a constant. Using the above information together with (2), we conclude that (14)-(15) hold. Due to (30) and the above,

$$
\left|\partial_{\tau} \partial_{\theta}^{k}\left(e^{2 P} Q_{\tau}\right)\right|=\left|\partial_{\theta}^{k+1}\left(e^{2 P-2 \tau} Q_{\theta}\right)\right| \leq C_{k} e^{-2 \tau} .
$$

Thus there is an $r \in C^{\infty}(I, \mathbb{R})$ such that (16) holds with $p$ replaced by $P$. Due to the estimates we have for the difference $P-p$, one can however prove that $(16)$ holds. Note that we know that $Q$ converges to a constant $q_{0}$ in any $C^{k}$ norm with an $e^{-2 \tau}$ bound. Let us compute

$$
\begin{aligned}
{\left[e^{2 p}\left(Q-q_{0}\right)\right](\tau, \cdot) } & =e^{2 p(\tau, \cdot)}\left(-\int_{\tau}^{\infty} Q_{\tau}(s, \cdot) d s\right)=-\int_{\tau}^{\infty} e^{2 p(\tau, \cdot)} Q_{\tau}(s, \cdot) d s \\
& =-\int_{\tau}^{\infty} e^{2(\tau-s)}\left(e^{2 p(s, \cdot)} Q_{\tau}(s, \cdot)-r\right) d s-\frac{r}{2}
\end{aligned}
$$


Thus

$$
\partial_{\theta}^{k}\left(e^{2 p}\left(Q-q_{0}\right)+\frac{r}{2}\right)(\tau, \cdot)=-\int_{\tau}^{\infty} e^{2(\tau-s)} \partial_{\theta}^{k}\left(e^{2 p(s, \cdot)} Q_{\tau}(s, \cdot)-r\right) d s .
$$

By (16), we get (17).

\section{Curvature Blow up on a Dense subset of the Singularity}

As we have already mentioned, we shall restrict our attention to the set $Y$ of smooth initial data, and given an element $\xi \in Y$, we shall associate a solution $x$ to (2)-(3) by specifying the initial data of $x$ at $\tau=0$ to be $\xi$. The set $Y$ can be given the topology of a complete metric space, cf. pp. 34-35 of [18]. Let $Y_{c}$ be the subset of $Y$ consisting of initial data for which the integral of the right hand side of (5) is zero. Let $p, q \in \mathbb{Q}$ be such that $0<q-p<1$ and let $n \in \mathbb{N}$. Define $\mathcal{C}_{p, q, n}$ to be the subset of $Y$ such that the corresponding solutions satisfy

$$
\left\|\frac{\rho(\tau, \cdot)}{\tau}-1\right\|_{C^{0}(K, \mathbb{R})} \leq n \tau^{-1}
$$

for all $\tau \geq 1$, where $K$ is the image of $[p, q]$ under the map that identifies multiples of $2 \pi, \rho$ is defined in $(23)$ and $z=\phi_{R D}(Q, P)$. In [16], we proved that $\rho(\tau, \theta) / \tau$ converges to $v_{\infty}(\theta)$. Consequently, (37) implies that $v_{\infty}=1$ on $K$.

Lemma 4.1. The sets $\mathcal{C}_{p, q, n}$ are closed in $Y$. Furthermore,

$$
\mathcal{G}=\cap_{p, q, n} \mathcal{C}_{p, q, n}^{c}
$$

is the set of initial data whose corresponding solutions have the property that $v_{\infty} \neq 1$ on a dense subset of the singularity. Finally, $\mathcal{C}_{p, q, n}^{c}$ is dense in $Y$ and $\mathcal{C}_{p, q, n}^{c} \cap Y_{c}$ is dense in $Y_{c}$.

Remark. By the results of [16], we conclude that the curvature is unbounded on a dense subset of the singularity if the initial data are in $\mathcal{G}_{c}$.

Proof. If $\xi_{k} \in \mathcal{C}_{p, q, n}$ converge to some $\xi \in Y$, then it is clear by the continuity of the map from initial data at one point in time to initial data at some other point in time that $\xi \in \mathcal{C}_{p, q, n}$. In other words, $\mathcal{C}_{p, q, n}$ is closed. Let $\xi \in \mathcal{G}$ and let $P, Q$ denote the corresponding solution. Consider the set of points $\mathcal{S}$ where $v_{\infty}=1$. Assume this set contains an open interval. Due to Proposition 1.1 and Theorem 1.1, we have (15) in some open interval $I$, after carrying out an inversion if necessary. Consequently,

$$
\left\|\frac{P(\tau, \cdot)}{\tau}-1\right\|_{C^{0}(I, \mathbb{R})} \leq n_{1} \tau^{-1},
$$

for $\tau \geq 1$ and some $n_{1}$. Note that if we let $z=\phi_{R D}(Q, P)$, then $z$ converges to $z_{0}=\left(q_{0}-i\right) /\left(q_{0}+i\right)$ on $I$ due to (17) and (22). Note also that $\rho$ defined in (23) is invariant under inversions, since $\phi_{R D} \circ \operatorname{Inv} \circ \phi_{R D}^{-1}(z)=-\bar{z}$. Since $z$ converges uniformly to $z_{0} \neq 1$, we conclude from (26) and (38) that (37) holds for $\tau \geq 1$ and some $n$. Since $I$ contains some interval $[p, q]$ with $p, q \in \mathbb{Q}, 0<q-p<1$, we conclude that $\xi \in \mathcal{C}_{p, q, n}$ for some $p, q, n$, i.e. that $\xi \notin \mathcal{G}$. Thus $\mathcal{S}^{c}$ is dense in $S^{1}$. On the other hand, if $\xi$ are initial data for which the corresponding solution has the property that the asymptotic velocity is different from 1 on a dense subset of the singularity, then clearly, $\xi \in \mathcal{C}_{p, q, n}^{c}$ for all $p, q, n$. 
What remains to be shown is that $\mathcal{C}_{p, q, n}^{c}$ is dense in $Y$ and that $\mathcal{C}_{p, q, n}^{c} \cap Y_{c}$ is dense in $Y_{c}$. Let $x$ be a solution corresponding to initial data in $Y$. We wish to prove that there is a sequence of solutions $x_{k}$ corresponding to initial data in $\mathcal{C}_{p, q, n}^{c}$ such that $x_{k} \rightarrow x$ in the $C^{\infty}$ topology of initial data. We shall use the notation $x_{T}$ to denote the initial data of $x$ for $\tau=T$ and similarly for other solutions. If $x_{T} \in \mathcal{C}_{p, q, n}^{c}$ for $T=0$, nothing remains to be proved, so we assume that this is not the case. We can thus assume that $x$ has the property that $v_{\infty}=1$ on $K$. After performing an inversion, if necessary, and restricting the interval to an open subinterval $I=\left(\theta_{0}-\epsilon, \theta_{0}+\epsilon\right)$ of $K$, if necessary, we get the asymptotics (14)-(17), cf. Theorem 1.1. We are now in a position to apply the results of [10] and [12]. This will be done in several steps. Each step is simple, but there are many of them, and for that reason, we shall write down the steps in detail.

Step 1: definition of $(\phi, \psi)$ and $(\tilde{\phi}, \tilde{\psi})$. The functions $r$ and $\phi$ are given by (14)-(17). Define $\psi=-e^{-2 \phi} r / 2$. Note that $\phi, \psi \in C^{\infty}(I, \mathbb{R})$ and that we have expansions of the form (12)-(13) with $v=1$ and $q=q_{0}$. Choose $\tilde{\psi}$ and $\tilde{\phi}$ to be smooth functions on $S^{1}$ such that they equal $\psi$ and $\phi$ respectively on $\left(\theta_{0}-9 \epsilon / 10, \theta_{0}+9 \epsilon / 10\right)$.

Step 2: definition of $\left(v_{k}, \phi_{k}, q_{k}, \psi_{k}\right)$. Let $\chi \in C^{\infty}\left(S^{1}, \mathbb{R}\right)$ be such that $\chi=0$ outside of $\left[\theta_{0}-\epsilon / 2, \theta_{0}+\epsilon / 2\right],|\chi| \leq 1 / 10$ and $\chi\left(\theta_{0}\right) \neq 0$. Define $\phi_{k}=\tilde{\phi}, \psi_{k}=\tilde{\psi}, q_{k}=q_{0}$ and $v_{k}=1+\chi / k$.

Step 3: definition of $y_{k}$ and $\tilde{x}$. By the existence result in [12], we know that there are unique solutions to the Gowdy equations corresponding to the data $\left(v_{k}, \phi_{k}, q_{k}, \psi_{k}\right)$ and $\left(1, \tilde{\phi}, q_{0}, \tilde{\psi}\right)$. Let $y_{k}$ and $\tilde{x}$ be the corresponding solutions and let us define $y_{k, T}$ and $\tilde{x}_{T}$ to be the initial data corresponding to these solutions for $\tau=T$.

Step 4: convergence of $y_{k}$ to $\tilde{x}$. To prove the convergence of $y_{k}$ to $\tilde{x}$, one can use the arguments of [12]. In that paper, the author proves that given suitable smooth data on the singularity, $(v, \phi, q, \psi)$ and a sequence of real analytic data converging to these data in $C^{\infty}$, then the real analytic solutions to the Gowdy equations constructed in [10] converge in the $C^{\infty}$ topology when restricted to a regular Cauchy hypersurface. The only reason the author restricted himself to considering a sequence of real analytic data on the singularity converging to smooth data was that until then existence was only known for real analytic data. Using exactly the same argument as in [12], but starting with a sequence of smooth initial data converging to smooth initial data, we get the conclusion that the corresponding initial data on a regular Cauchy surface converge.

Step 5: uniqueness. By a uniqueness result proven in [12], $y_{k, T}=\tilde{x}_{T}$ on

$$
\left(\theta_{0}-8 \epsilon / 10, \theta_{0}-3 \epsilon / 4\right) \cup\left(\theta_{0}+3 \epsilon / 4, \theta_{0}+8 \epsilon / 10\right)
$$

for $T$ large enough. Furthermore, $x_{T}$ coincides with $\tilde{x}_{T}$ on $\left(\theta_{0}-8 \epsilon / 10, \theta_{0}+8 \epsilon / 10\right)$ for $T$ large enough.

Step 6: definition of $x_{k}$. Fix some large $T$ such that the uniqueness of step 5 holds. Let the initial data for $x_{k}$ at $T, x_{k, T}$, coincide with $y_{k, T}$ on $\left[\theta_{0}-3 \epsilon / 4, \theta_{0}+3 \epsilon / 4\right]$, and $x_{k, T}=x_{T}$ outside of this set. Then $x_{k}$ has the asymptotics of $y_{k}$ on the interval $\left(\theta_{0}-\epsilon / 2, \theta_{0}+\epsilon / 2\right)$.

Step 7: convergence of $x_{k, T}$ to $x_{T}$. Note that by step 5 and $6, x_{k, T}-x_{T}$ is zero outside of $\left[\theta_{0}-3 \epsilon / 4, \theta_{0}+3 \epsilon / 4\right]$ and equals $y_{k, T}-\tilde{x}_{T}$ inside this interval. By step 4 , we get convergence of $x_{k, T}$ to $x_{T}$ with respect to the $C^{\infty}$ topology. Since $x_{k}$ does 
not have asymptotic velocity identically equal to 1 in $I$, we conclude that $\mathcal{C}_{p, q, n}^{c}$ is dense in $Y$

Step 8: $\mathcal{C}_{p, q, n}^{c} \cap Y_{c}$ is dense in $Y_{c}$. Assume the integral of the right hand side of (5) is zero. Then we want the same to be true for the approximations $x_{k}$ constructed above. Consider

$$
j_{\epsilon}(T)=\int_{\theta_{0}-3 \epsilon / 4}^{\theta_{0}+3 \epsilon / 4}\left(P_{\theta} P_{\tau}+e^{2 P} Q_{\theta} Q_{\tau}\right)(T, \theta) d \theta .
$$

Since we have asymptotic expansions of the form (14)-(17), we conclude that

$$
\lim _{T \rightarrow \infty} j_{\epsilon}(T)=\phi\left(\theta_{0}+3 \epsilon / 4\right)-\phi\left(\theta_{0}-3 \epsilon / 4\right) .
$$

Let $\left(Q_{k}, P_{k}\right)=x_{k}$. Due to the equations, the integral of the right hand side of (5) is always conserved. To prove that it is zero, we need in other words only prove that the integral converges to zero as $T$ tends to infinity. Since we know that $x_{k, T}=x_{T}$ outside of $\left[\theta_{0}-3 \epsilon / 4, \theta_{0}+3 \epsilon / 4\right]$, all we need to prove is that

$$
j_{k, \epsilon}(T)=\int_{\theta_{0}-3 \epsilon / 4}^{\theta_{0}+3 \epsilon / 4}\left(P_{k \theta} P_{k \tau}+e^{2 P_{k}} Q_{k \theta} Q_{k \tau}\right)(T, \theta) d \theta .
$$

has the property that

$$
\lim _{T \rightarrow \infty} j_{k, \epsilon}(T)=\phi\left(\theta_{0}+3 \epsilon / 4\right)-\phi\left(\theta_{0}-3 \epsilon / 4\right)
$$

for all $k$. However,

$$
\lim _{T \rightarrow \infty} j_{k, \epsilon}(T)=\phi\left(\theta_{0}+3 \epsilon / 4\right)-\phi\left(\theta_{0}-3 \epsilon / 4\right)+\int_{\theta_{0}-3 \epsilon / 4}^{\theta_{0}+3 \epsilon / 4} \frac{1}{k} \chi \phi_{\theta} d \theta
$$

In other words, we need to choose $\chi$ so that it is orthogonal to $\phi_{\theta}$. This is not a problem.

\section{REFERENCES}

[1] Berger B, Isenberg J and Weaver M 2001 Oscillatory approach to the singularity in spacetimes with $T^{2}$ isometry Phys. Rev. D 64084006

[2] Berger B and Garfinkle D 1998 Phenomenology of the Gowdy universe on $T^{3} \times \mathbb{R}$ Phys. Rev. D 57 1767-77

[3] Berger B and Moncrief V 1993 Numerical investigation of cosmological singularities Phys. Rev. D. $484676-4688$

[4] Chae M and Chruściel P T 2004 On the dynamics of Gowdy space times Commun. Pure Appl. Math. 57 1015-1074

[5] Chruściel P T 1990 On spacetimes with $U(1) \times U(1)$ symmetric compact Cauchy surfaces Ann. Phys. NY 202 100-50

[6] Chruściel P T and Lake K 2004 Cauchy horizons in Gowdy space times Class. Quantum Grav. 21 S153-S170

[7] Gowdy R H 1974 Vacuum spacetimes with two-parameter spacelike isometry groups and compact invariant hypersurfaces: Topologies and boundary conditions Ann. Phys. NY 83 203-41

[8] Grubišić B and Moncrief V 1993 Asymptotic behaviour of the $T^{3} \times \mathbb{R}$ Gowdy space-times Phys. Rev. D 47 2371-82

[9] Isenberg J and Moncrief V 1990 Asymptotic behaviou of the gravitational field and the nature of singularities in Gowdy space times Ann. Phys 199 84-122

[10] Kichenassamy S and Rendall A 1998 Analytic description of singularities in Gowdy spacetimes Class. Quantum Grav. 15 1339-55

[11] Moncrief V 1981 Global properties of Gowdy spacetimes with $T^{3} \times \mathbb{R}$ topology Ann. Phys. NY 132 87-107 
[12] Rendall A 2000 Fuchsian analysis of singularities in Gowdy spacetimes beyond analyticity Class. Quantum Grav. 17 3305-16

[13] Rendall A and Weaver M 2001 Manufacture of Gowdy spacetimes with spikes Class. Quantum Grav. 18 2959-76

[14] Ringström H 2004 On Gowdy vacuum spacetimes Math. Proc. Camb. Phil. Soc. 136 485-512

[15] Ringström H 2004 Asymptotic expansions close to the singularity in Gowdy spacetimes $A$ Spacetime Safari: Essays in honour of Vincent Moncrief, Special issue of Class. Quantum Grav., Eds. B Berger and J Isenberg 21 S305-S322

[16] Ringström H 2004 On the concept of an asymptotic velocity in $T^{3}$ Gowdy spacetimes Preprint

[17] Ringström H 2004 Strong cosmic censorship in $T^{3}$-Gowdy spacetimes Preprint

[18] Rudin W 1991 Functional Analysis (McGraw-Hill)

Max-Planck-Institut für Gravitationsphysik, Am Mühlenberg 1, D-14476 Golm, Germany, Present address: Department of Mathematics, KTH, 10044 Stockholm, Sweden. 